\title{
Comparaison of the Optical Anisotropy of Corrugated and Non- Corrugated (311) Superlattices
}

\author{
Abdelaziz Hallaoui ${ }^{1, *}$, Hafida Essaoudi ${ }^{2}$, and Abdelouahed Essahlaoui ${ }^{1}$ \\ ${ }^{I}$ (Engineering Sciences Laboratory (LSI), Multidisciplinary Faculty of Taza, \\ Sidi Mohamed Ben Abdellah University, B.P. 1223 Taza gare, Taza Morocco) \\ ${ }^{2}$ (Faculty of Sciences, Moulay Ismail University, B.P.1120 Zitoune, Meknes Morocco)
}

\begin{abstract}
We report the electronic and optical properties of corrugated and non-corrugated (311) $(G a A s) /(A l A s)$ superlattices using the tight-binding method. These systems grown by molecular beam epitaxy on (311) GaAs oriented substrates. We present the valence and conduction band dispersion relations (band structures) for the corrugated (311) (GaAs) ${ }_{16}(\text { AlAs })_{16}$ superlattice. We have studied the probability densities of the lowest conduction band and highest valence band interband transition probabilities of the non-corrugated (311) $(G a A s)_{8} /(A l A s)_{8}$ superlattice and the corrugated (311) $(G a A s)_{16} /(A l A s)_{16}$ superlattice. We have also computed the interband transition probabilities for these systems. We found that the anisotropy rate of the interband transitions for the corrugated superlattice is larger than for the non-corrugated superlattice one. We concluded that whatever the type of superlattice studied here, the optical anisotropy is reduced when the light polarization pass from the [-2,3,3] direction to [0,-1,1] direction for the transitions from the heavy-hole-like states. The opposite situation occurs for the transition from the light-hole and split-off-like states.
\end{abstract}

Keywords: Anisotropy rate - Corrugated superlattice - Interband transition probability - Non-corrugated superlattice - Probability density

\section{Introduction}

For two decades, many experimental and theoretical studies have been carried out to study the one dimensional (1D) semiconductor nanostructures because of their unique electronic and optical properties [1]. Among them the GaAs/AlAs (311) superlattices grown by molecular beam epitaxy (MBE) and who have a corrugated interface with gives rise to quantum wire-like structure [2-7]. This nanostructure find a great spectrum of technological application in nanoscale electronic and optoelectronic devices : spintronic devices [8,9], switching devices [10], infrared photodetectors [11-13], field-effect transistors [14,15], solar cells [16], lasers [17] etc...

Recently, V.G. Dorogan et al [18] reported very impressive result of optical anisotropy of the $\mathrm{In}_{0.40} \mathrm{Ga}_{0.60} \mathrm{As}$ grown by MBE on GaAs (311)A and GaAs (331)A substrates. They investigated this sample by linearly polarized dependence and they found presents the linearly polarized photoluminescence (PL) spectra, with angular dependence. They found also that a strong polarization of electron-heavy-hole transition along the [-233] and [01-1] (for (311)A sample) and [-110] and [11-6] (for (331)A sample) directions can be observed which is caused by the anisotropy of these systems.

C. Jouanin and al [19]. report a calculation of the electronic properties of a oriented GaAs/AlAs (311) superlattices through the Brillouin zone using realistic values of the parameters in a tight - binding Hamiltonian. Optical properties of (311) corrugated superlattice are studied also by C. Jouanin et al [20] and the calculated interband transitions account for the photoluminescence data.

The interest of this computation for the corrugated and non-corrugated $(3,1,1)$ superlattices $[\mathbf{1 9 , 2 0}]$ which is based on the tight binding method and $\mathrm{sp}^{3} \mathrm{~s}^{*}$ model lies in the obtaining:

Firstly the electronic properties of the two kind of superlattices. The predominant heavy-hole or light hole character of two upper valence bands, the $\Gamma$ or $\mathrm{X}$ type two lowest conduction states and the confinement of valence and conduction energy levels.

Secondly, the optical anisotropy of the allowed transition of the corrugated and non-corrugated $(3,1,1)$ superlattices. The study of the anisotropy come from the corrugation because the anisotropy of the corrugated superlattice is more important than for non-corrugated $(3,1,1)$-grown superlattice.

In this paper, we will calculate a band structure of the (311) $(\mathrm{GaAs})_{16} /(\mathrm{AlAs})_{16}$ corrugated superlattice. We will also report the probability densities of the conduction and valence states for the non-corrugated and corrugated (311) (GaAs)/(AlAs) superlattices and optical transition matrix element of this nanostructures. Finaly we will compare their optical anisotropy. 


\section{Presentation Superlattice}

Over the last years, the the $\mathrm{GaAs} /(\mathrm{GaAl})$ nanostructures have been intensively studied and detailed understanding of the electronic and optical properties of quantum wells and superlattices built up with these compounds is due to high quality samples with thin layers. The (100)-oriented structures which were the first obtained structures were the object of most of these investigations. Growth along other crystallographic directions has produced interest in less symmetric surfaces like (110) and has given good quality samples. Molecular beam epitaxy on high Miller-index surfaces has allowed the achievement of high-quality structures and (311) superlattices are available for experimental investigations of their optical properties. A method made it possible to obtain quantum wires by direct epitaxy of superlattice structures with periodic corrugation of the interface [3]. The breaking up of the (311) surfaces into (311) facets with lower surface energy for certain growth conditions gives rise to these structures. The geometry in question gives rise to a lateral confinement and for thin semiconductor layers, quantum wires are formed.

\section{Computation Method}

We use a tight-binding approach, to describe the bulk crystal. The interest of this computational method rests in the microscopic description of the materials from the atomic interactions between anions and cations which gives the right crystal symmetry and avoids preliminary hypothesis on the origin of the superlattice electronic states. To obtain the best possible values for the energies and effective masses at high-symmetry points in Brillouin zone, e.g. $\Gamma$ and $\mathrm{X}$ conductions edges, we use $\mathrm{sp}^{3} \mathrm{~s}^{*}$ basis taking into account spin-orbit interactions [21] and fitting the Hamiltonian matrix elements. We have included the second-nearest-neighbour interactions between cation and anion p orbitals in the manner described in Ref. [22], in order to get some dispersion for bulk electronic bands along the X-W symmetry line. We take the value of valence band discontinuity between GaAs and AlAs equal to 35\% of the direct-band-gap difference. Near the interfaces, the environment is different from that of the massive material and can modify the value of the tight-binding parameters and different geometrical situations occur in corrugated superlattice. The utilisation of means values of the two bulk parameters takes into account these various configurations. The electronic band structure is not sensitive to this hypothesis, because this work does not concern the very short-period superlattices. The effect of the slight lattice mismatch $(0,16 \%)$ between GaAs and AlAs are neglected for the considered structures. The unit cell of corrugated superlattices is two dimensional and the tight binding basis is very large containing $320 \mathrm{~N}$ local orbitals for a superlattice of $\mathrm{N}$ layers along the growth direction [311]. The electronic energies are computed by direct diagonalisation or by alternative modes more adapted to handle large dimensional matrices as the the spectral moments method [23].

4.1. Brillouin zone - Band structure.

\section{Results And Discussion}

\subsubsection{Brillouin zone of a (311) corrugated superlattice.}

The new axes corresponding to the $(3,1,1)$ interfaces are:

$$
\begin{aligned}
& \vec{x}^{\prime}=\frac{1}{\sqrt{2}}(0,1,-1) \\
& \vec{y}^{\prime}=\frac{1}{\sqrt{2}}(-2,3,3) \\
& \vec{z}^{\prime}=\frac{1}{\sqrt{2}}(3,1,1)
\end{aligned}
$$

The fundamental translation vectors of direct lattice are given by :

$$
\begin{aligned}
& \vec{a}_{1}^{\prime}=\frac{a}{8 \sqrt{2}} \vec{x}^{\prime} \\
& \vec{a}_{2}^{\prime}=\frac{a \sqrt{22}}{2} \vec{y}^{\prime} \\
& \vec{a}_{3}^{\prime}=\frac{a}{2}\left(\frac{6}{\sqrt{22}} \vec{y}^{\prime}+\frac{2 N}{\sqrt{11}} \vec{z}^{\prime}\right)
\end{aligned}
$$

So The fundamental translation vectors of reciprocal lattice are:

$\vec{b}_{1}^{\prime}=\frac{\pi}{2 \sqrt{2 a}} \vec{x}^{\prime}$

$\vec{b}_{2}^{\prime}=\frac{2 \pi}{\sqrt{11 a}}\left(\sqrt{2} \vec{y}^{\prime}-\frac{3}{N} \vec{z}^{\prime}\right)$

$\vec{b}_{3}^{\prime}=\frac{2 \pi \sqrt{11}}{N a} \vec{z}^{\prime}$ 


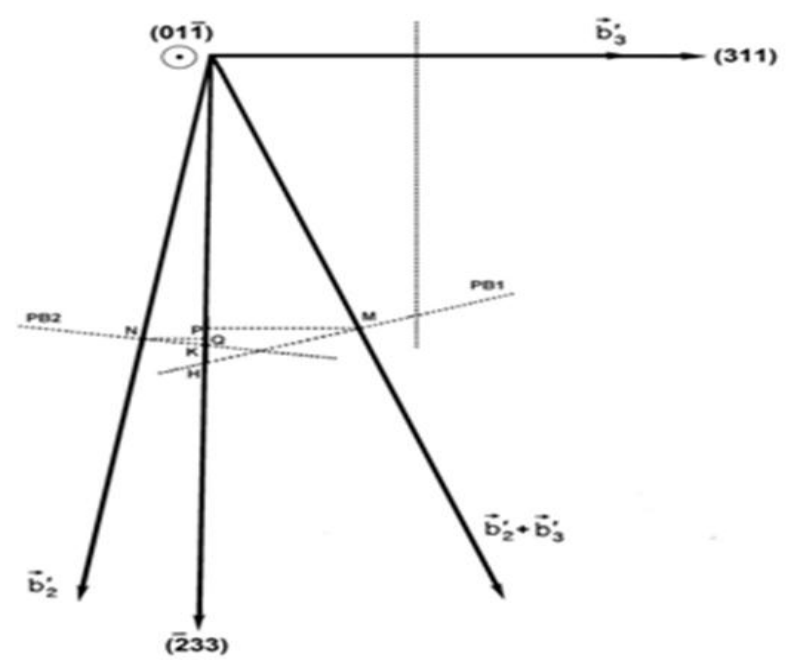

Figure 1: Brillouin zone projection on the $(0,1,-1)$ plan of the $(3,1,1)$ corrugated superlattice

The bisectors plans as shown in figure 1 limit the Brillouin zone along the $(0,1,-1)$ and $(3,1,1)$ directions at the points $\bar{X}$ and $\bar{Z}$. The coordinates of this points are, respectively, $\frac{\pi}{8 a}(0,1,-1)$ et $\frac{\pi}{N a}(3,1,1)$.

The bisector plans PB1 and PB2 contain the points M and N. They intersect with the y direction, respectively, at the points $\mathrm{H}$ and $\mathrm{K}$. The distances $\mathrm{OH}$ and $\mathrm{OK}$ are given by:

$$
\begin{aligned}
& O H=\frac{O M}{\cos \beta}=\frac{2 \pi}{a} \frac{1}{\sqrt{22}}\left(1+\frac{32}{N^{2}}\right) \text {, where } \cos \beta=\left(1+\frac{32}{N^{2}}\right)^{-1 / 2} \\
& O K=\frac{O N}{\cos \alpha}=\frac{2 \pi}{a} \frac{1}{\sqrt{22}}\left(1+\frac{9}{2 N^{2}}\right) \text {, where } \cos \alpha=\left(1+\frac{9}{2 N^{2}}\right)^{-1 / 2} \\
& \quad \alpha \text { and } \beta \text { are respectively the angles between } \vec{y} \text { direction and respectively } \vec{b}_{2}^{\prime} \text { and } \vec{b}_{2}^{\prime}+\vec{b}_{3}^{\prime}
\end{aligned}
$$
directions.

The distance $\mathrm{OK}$ is lower than the distance $\mathrm{OH}$ when $\mathrm{N}$ is small, however it's identical with the distance $\mathrm{OH}$ when $\mathrm{N}$ is infinite. Thus it is the point $\bar{Y}(=\mathrm{K}=\mathrm{H})$ which limit the Brillouin zone along the $\vec{y}$ direction. Its coordinates are: $\frac{\sqrt{2} \pi}{a}(-2,3,3)^{\text {. }}$

\subsubsection{Band structure of the (311) (GaAs) ${ }_{16}$ (AlAs) ${ }_{16}$ corrugated superlattice}

We plot respectively in figures 2,3 and 4 the six highest valence-suband energies and six lowestsuband energies along, respectively, the three directions $[0,1,-1],[3,1,1]$ and $[-2,3,3]$.

We have given respectively in figures $2 . \mathrm{b}$ and $3 . \mathrm{b}$, the dispersion along the two respectively directions $[0,1,-1]$ and $[3,1,1]$ is small both for the first and third valence states, but it is more important for the second valence state. Along the $[-2,3,3]$ direction, the dispersion is important for the three first valence states as shown in figure 4.b.

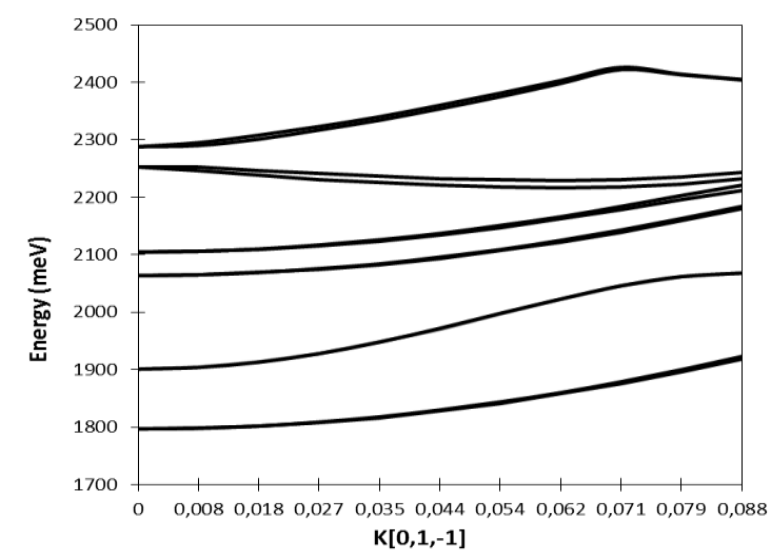

Figure 2.a: Conduction band structure of the $(3,1,1)(\mathrm{GaAs})_{16} /(\mathrm{AlAs})_{16}$ corrugated superlattice along the $[0,1,-1]$ direction 


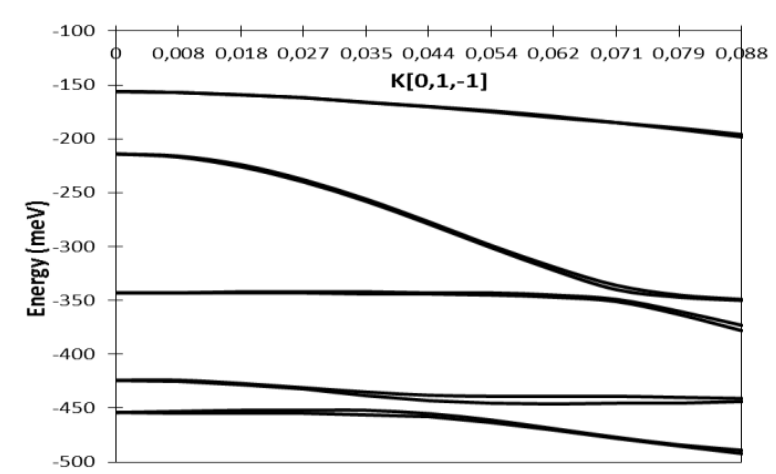

Figure 2.b: Valence band structure of the $(3,1,1)(\mathrm{GaAs})_{16} /(\mathrm{AlAs})_{16}$ corrugated superlattice along the $[0,1,-1]$ direction

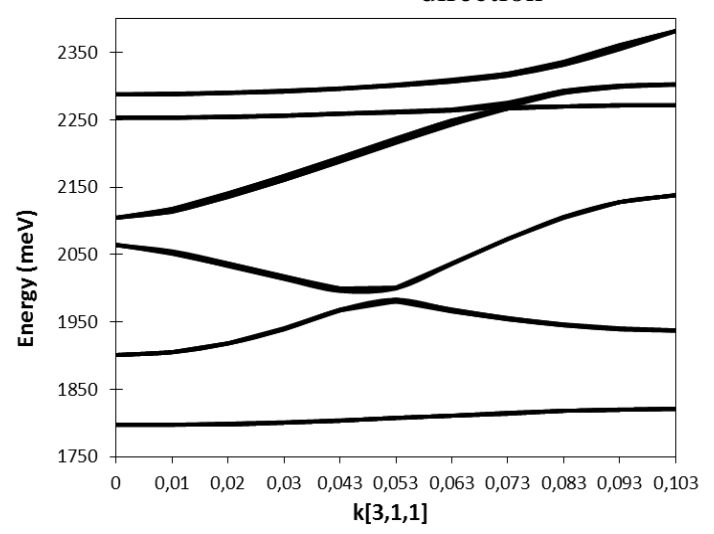

Figure 3.a: Conduction band structure of the $(3,1,1)(\mathrm{GaAs})_{16} /(\mathrm{AlAs})_{16}$ corrugated superlattice along the $[3,1,1]$ direction

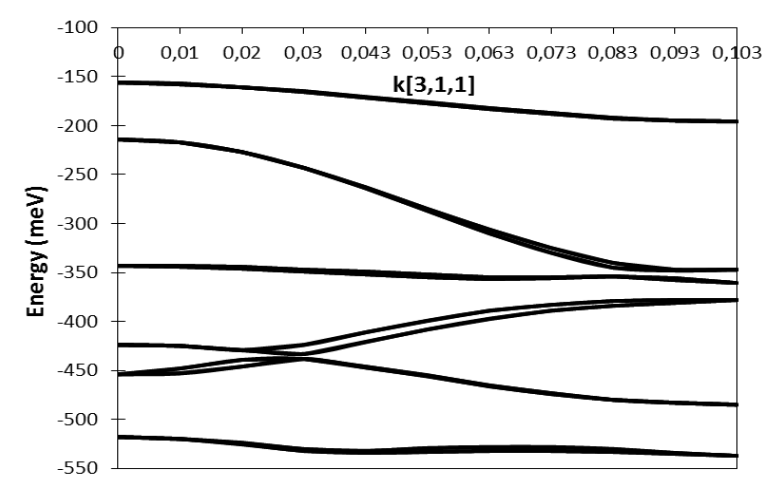

Figure 3.b: Valence band structure of the $(3,1,1)(\mathrm{GaAs})_{16} /(\mathrm{AlAs})_{16}$ corrugated superlattice along the $[3,1,1]$ direction

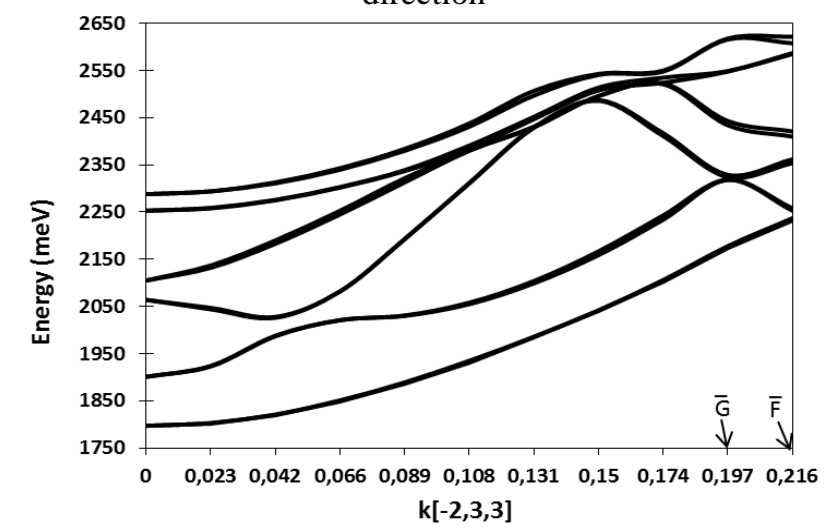

Figure 4.a: Conduction band structure of the $(3,1,1)(\mathrm{GaAs})_{16} /(\mathrm{AlAs})_{16}$ corrugated superlattice along the [$2,3,3]$ direction 


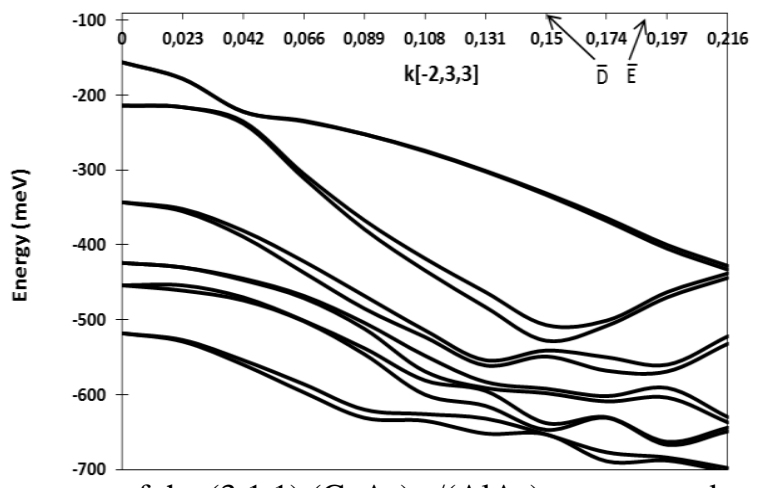

Figure 4.b: Valence band structure of the $(3,1,1)(\mathrm{GaAs})_{16} /(\mathrm{AlAs})_{16}$ corrugated superlattice along the $[-2,3,3]$ direction

As shown in figures 2.a, 3.a and 4.a, the dispersion is important for the second conduction state along, respectively, the three directions $[0,1,-1],[3,1,1]$ and $[-2,3,3]$.

Of same, along the two directions $[0,1,-1]$ and $[-2,3,3]$ (figures 2 .a and 4.a), the dispersion is important for the first conduction state, but it's small along the $[3,1,1]$ direction (figure $3 . a$ ).

The minimum of the first valence state $(-433 \mathrm{meV})$ occurs at the point which limits the Brillouin zone along the $[-2,3,3]$ direction. The lowest energy of the the second valence state $(-528 \mathrm{meV})$ along the $[-2,3,3]$ direction occurs at the point $\bar{D}$ which is 0.15 (in $2 \pi /$ a unit) away from the point $\bar{\Gamma}$. We remark that the spinorbit splitting due the lack inversion symmetry of the second valence state is important along this direction. The two spin components of this state are separated by $21 \mathrm{meV}$ at the point $\bar{D}$. Moreover at the point $\bar{E}$ of the [$2,3,3$ ] direction which is 0.192 (in $2 \pi /$ a unit) away from the point $\bar{\Gamma}$, the energy of the third valence state is minimal. The spin-orbit splitting of this state is also important in the $[-2,3,3]$ direction. That spin-orbit splitting is equal to $11 \mathrm{meV}$ at the point $\bar{E}$. The maximum of the two first conduction states occurs respectively, at the points $\bar{F}$ and $\bar{G}$ of the $[-2,3,3]$ direction which are respectively, away 0.216 and 0.197 (in $2 \pi /$ a unit) from the point $\bar{\Gamma}$. The maximums and minimums of respectively, the three first valence states and the two first conduction states occurs at the point $\bar{\Gamma}$. So for the corrugatted (311) (GaAs) $16^{/(\mathrm{AlAs})} 16$ superlattice, the gap is direct.

\subsection{Probability densities of the conduction and valence states for the non-corrugated and corrugated (311) $($ GaAs)/(AlAs) superlattices. 4.2.1. Non-corrugated (311) $(\text { GaAs })_{8} /(\text { ALAs })_{8}$ superlattice.}

For the (311) non-corrugated $(\mathrm{GaAs})_{8} /(\mathrm{ALAs})_{8}$ superlattice, the first and third valence states are heavyhole like. Their energies are respectively -159 and -519 mev. However, the second valence state has a predominant light-hole character. His energy is equal to $-228 \mathrm{meV}$.

For the first conduction state, the energy at the point $\bar{Y}(1908 \mathrm{meV})$ is lower than the energy at the point $\bar{\Gamma}(1794 \mathrm{meV})$. To obtain, the probability densities of the valence and conduction states, we compute the squared total amplitude of the cation and anion orbital components. In figures 5.a and 5.b, we plot respectively, the probability densities of the first and second conduction states at $\bar{Y}$ and $\bar{\Gamma}$. Of same, the probability densities of the first three valence states at the point $\bar{\Gamma}$ are represented, respectively, in figures 6.a, 6.b and 6.c.

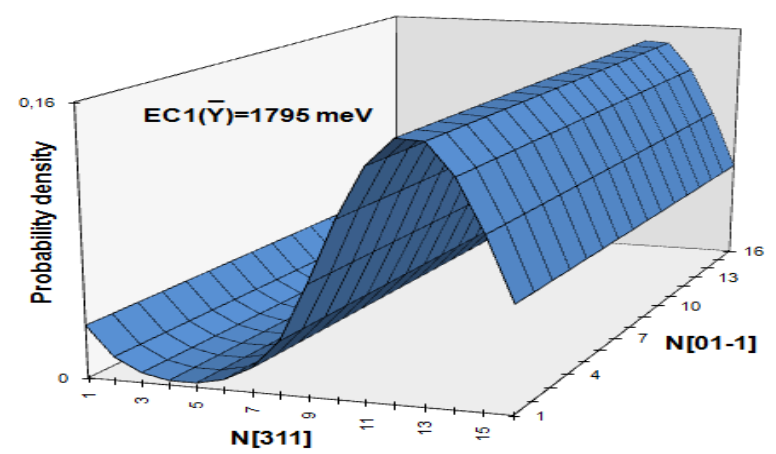

Figure 5.a: Probability density of the $\bar{Y}$ lowest conduction band for the $(\mathrm{GaAs})_{8} /(\mathrm{AlAs})_{8}(311)$ non-corrugated superlattice 


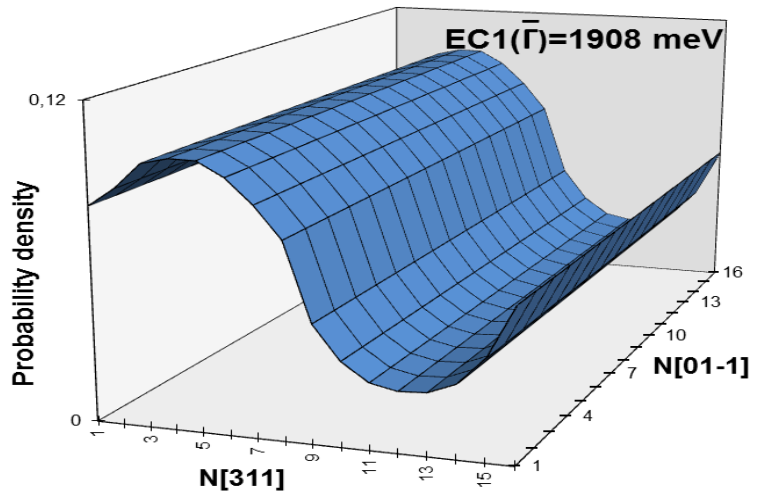

Figure 5.b: Probability density of the $\bar{\Gamma}$ lowest conduction band for the $(\mathrm{GaAs})_{8} /(\mathrm{AlAs})_{8}(311)$ noncorrugated superlattice

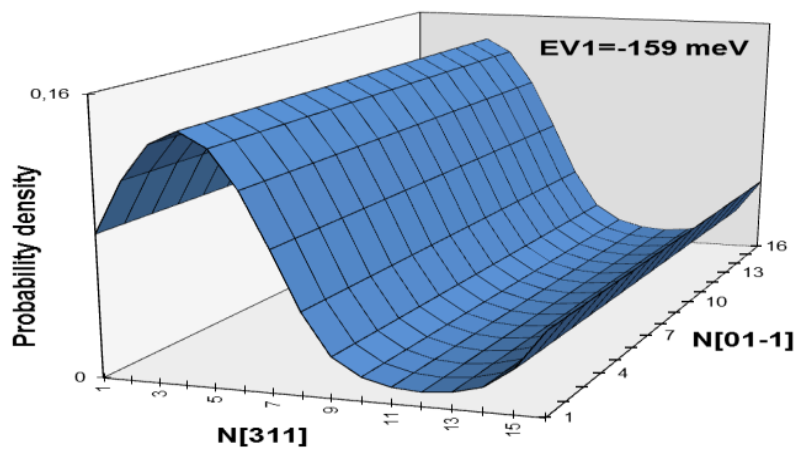

Figure 6.a: Probability density of the $\bar{\Gamma}$ highest valence band for the $(\mathrm{GaAs})_{8} /(\mathrm{AlAs})_{8}(311)$ non-corrugated superlattice

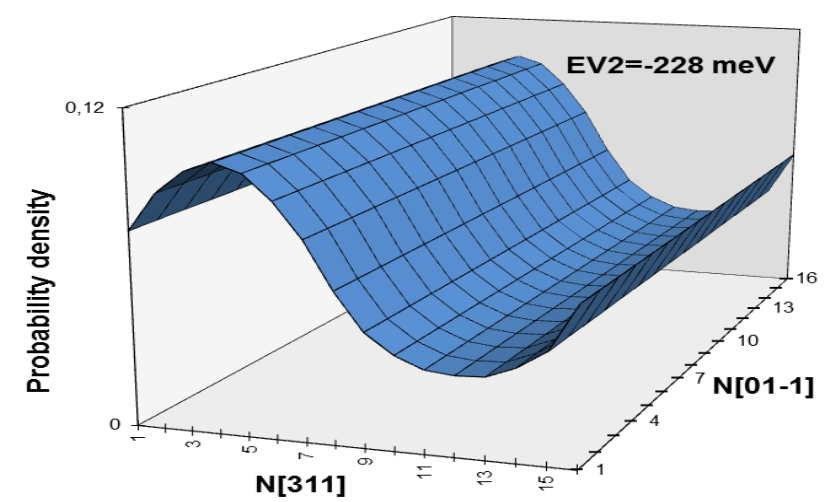

Figure 6.b: Probability density of the $\bar{\Gamma}$ second highest valence band for the $(\mathrm{GaAs})_{8} /(\mathrm{AlAs})_{8}(311)$ noncorrugated superlattice

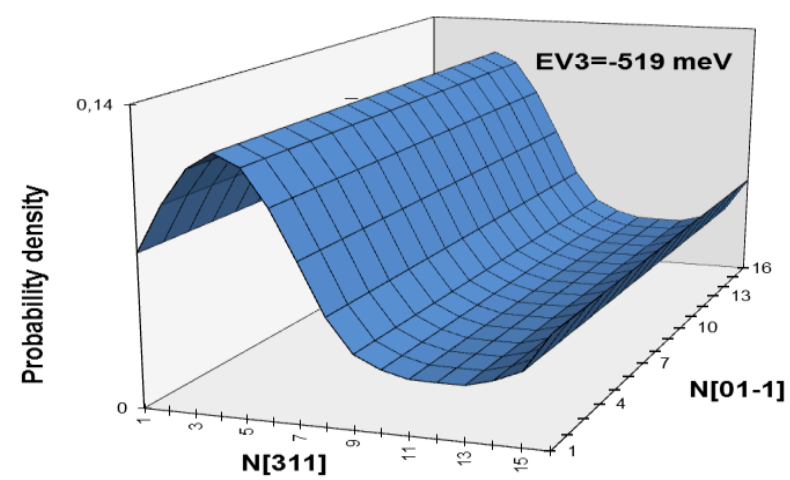

Figure 6.c: Probability density of the $\bar{\Gamma}$ third highest valence band for the $(\mathrm{GaAs})_{8} /(\mathrm{AlAs})_{8}(311)$ noncorrugated superlattice 
The lowest electron state at the point $\bar{Y}$ is strongly localized in AlAs layers and has maximums at the center of these layers. However, the maximum of the probability densities of the lowest conduction state at the point $\bar{\Gamma}$ occurs at the center of the GaAs layers. This state is strongly localized in GaAs layers.

The three first valence states are localized in GaAs layers, the first and third valence states has an odd parity, but the second valence state has an even parity.

\subsubsection{Corrugated (311) (GaAs) ${ }_{16}^{/(A L A s)}{ }_{16}$ superlattice}

In order to show the effect of the confinement on the electronic wave functions, we have plotted in the figures 7.a and 7.b, the probability densities of the two lowest conduction bands for the (GaAs) ${ }_{16} /(\mathrm{ALAs})_{16}$ (311) corrugated superlattice.

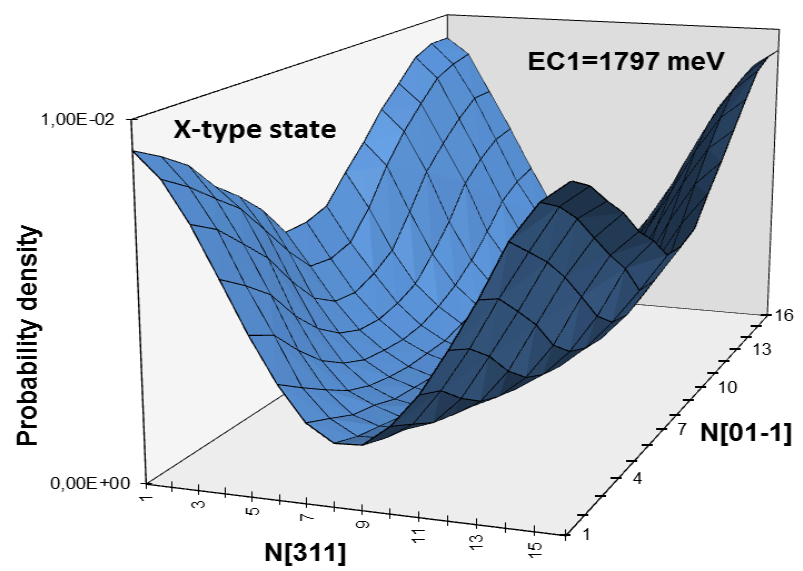

Figure 7.a: Probability density of the $\bar{\Gamma}$ lowest conduction band for the $(\mathrm{GaAs})_{16} /(\mathrm{AlAs})_{16}(311)$ corrugated superlattice

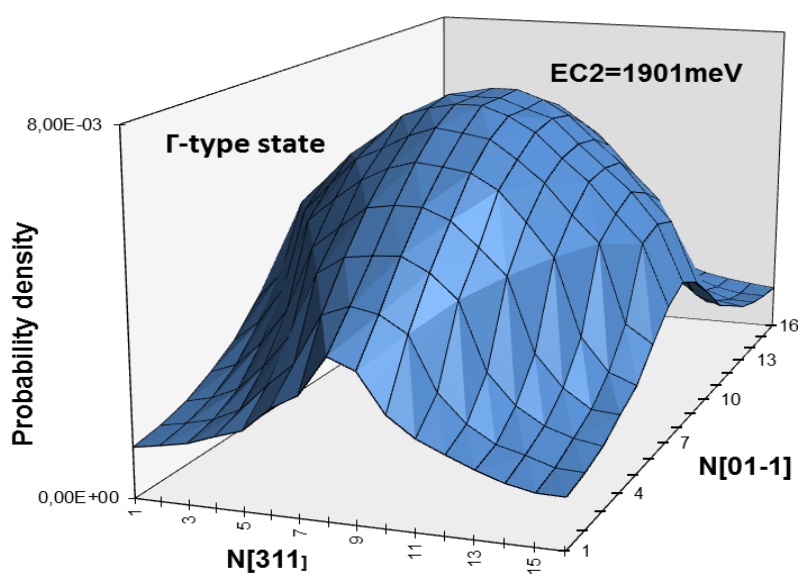

Figure 7.b: Probability density of the $\bar{\Gamma}$ second lowest conduction band for the GaAs) ${ }_{16} /(\mathrm{AlAs})_{16}(311)$ corrugated superlattice

Because the diagonalization of the hamiltonien matrix allow to obtain all contributions, we give the squared total amplitude of the cation and anion orbitals components. The energy of the lowest electron state is $1797 \mathrm{meV}$. This state is strongly confined in AlAs layers and has peaks at the center of these layers. However, the maximum of the probability density of the second lowest state occurs at the center of the GaAs layers, and this state is strongly confined in GaAs layer.

The energy of this state is $1901 \mathrm{meV}$. The effect of the corrugation on the electronic wave functions of these two states is well marked and electrons are mainly confined in the one or other layer. In the following, we discuss the two highest valence subbands which are presented in figures 8.a and 8.b. At $\bar{\Gamma}$ the two upper valence bands are localized in GaAs layers, their energy are $-156 \mathrm{meV}$ and $-214 \mathrm{meV}$. The highest energy state has predominant heavy-hole character. The confinement is less strong than in the (100) superlattice with the same thickness. However, this effect is less pronounced than for (111) grown superlattices. The second energy state is light-hole like and its energy is nearly the same as for the other growth directions because of the isotropy of the light-hole band. 


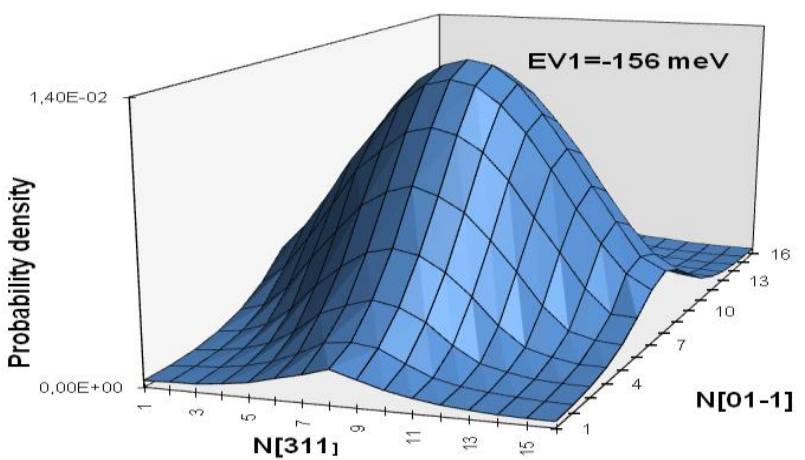

Figure 8.a: Probability density of the $\bar{\Gamma}$ highest valence band for the (GaAs) ${ }_{16} /(\mathrm{AlAs})_{16}(311)$ corrugated superlattice

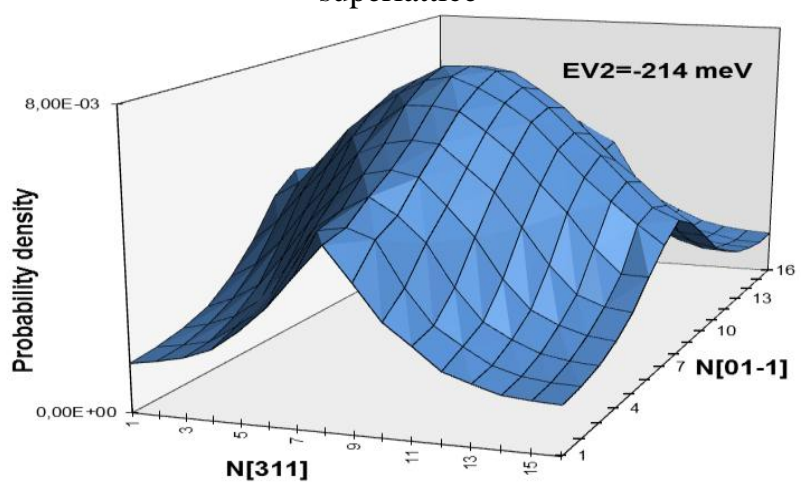

Figure 8.b: Probability density of the $\bar{\Gamma}$ second highest valence band for the $(\mathrm{GaAs})_{16} /(\mathrm{AlAs})_{16}(311)$ corrugated superlattice

\subsection{Optical anisotropy of the non-corrugated $(\mathrm{GaAs})_{8} /(\mathrm{AlAs})_{8}(311)$ and corrugated $(\mathrm{GaAs})_{16} d(\mathrm{AlAs})_{16}(311)$} superlattices

We have calculated the optical properties of the two superlattices: the first one is the $(\mathrm{GaAs})_{16} /(\mathrm{ALAs})_{16}$ (311) corrugated superlattices, the second is the $(\mathrm{GaAs})_{8} /(\mathrm{ALAs})_{8}$ (311) non-corrugated superlattices. In figure 9 we show the curves which the parametric equation is given by:

$I[-2,3,3]=I(\theta) \cos \theta$

$I_{[0,1,-1]}=I(\theta) \sin \theta$

Where $I(\theta)$ and $\theta$ are the parameters of theses curves.

$\theta$ is the angle between the light polarization orientation and $[-2,3,3]$ direction. $I(\theta)$ is the transition interband probabilities when the light is polarized along the direction which has the angle $\theta$ with the $[-2,3,3]$ direction. For the corrugated superlattice the two lowest transition in energy $\bar{\Gamma}_{1 v}-\bar{\Gamma}_{2 c}$ and $\bar{\Gamma}_{2 v}-\bar{\Gamma}_{2 c}$ appearing in the calculated spectra originate from the upper valence bands which have, respectively, a heavy-hole and light-hole dominant character while the finale state is always the second lowest-lying $\bar{\Gamma}$ conduction band.

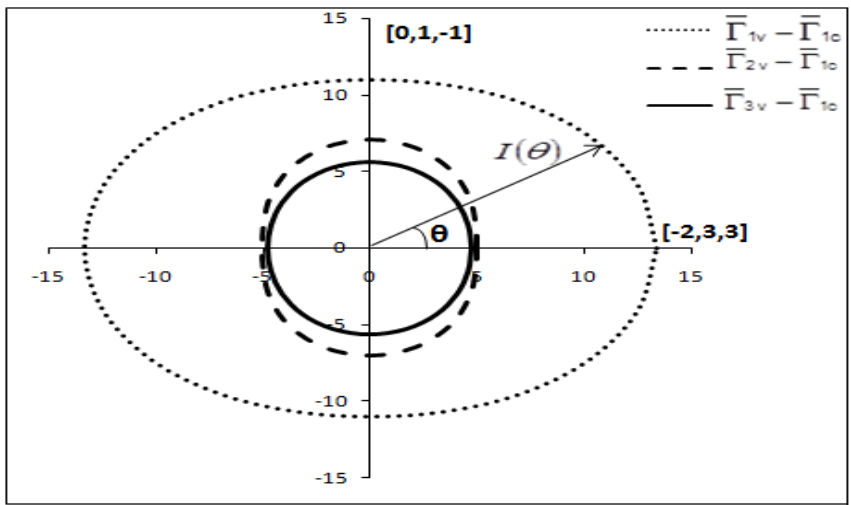

Figure 9.a: Parametrical curves which show the transition interband probabilities in the (311) non-corrugated superlattices 


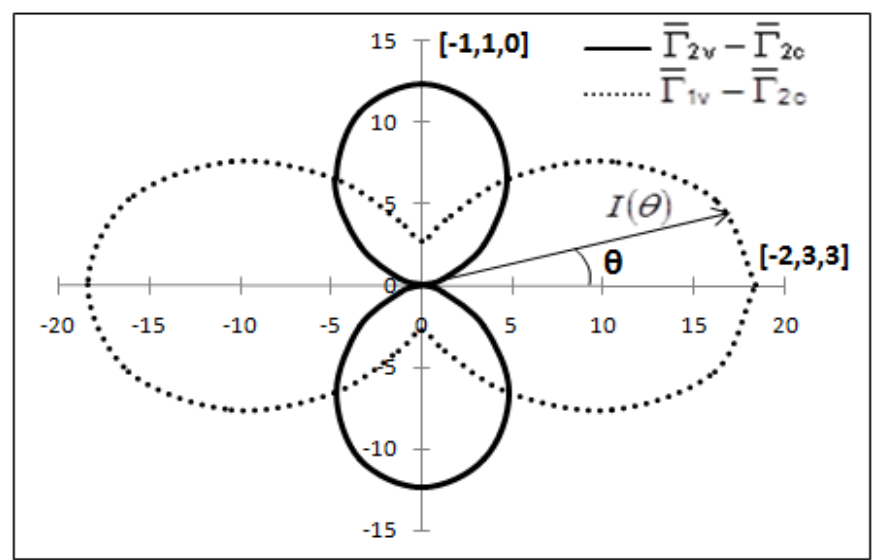

Figure 9.b: Parametrical curves which show the transition interband probabilities in the (311) corrugated superlattices

For the non-corrugated superlattice there are three lowest transitions in energy $\bar{\Gamma}_{1 v}-\bar{\Gamma}_{1 c}, \bar{\Gamma}_{2 v}-\bar{\Gamma}_{1 c}$ and $\bar{\Gamma}_{3 v}-\bar{\Gamma}_{1 c}$ which have, respectively, a heavy-hole, light-hole and split-off dominant character. The energy of these transitions for the two kinds of superlattices is given in the Tables 1 and 2 below:

Table 1: Inerband transition energies (in meV) of the non-corrugated superlattice

\begin{tabular}{|l|c|c|c|}
\hline Transition & $\bar{\Gamma}_{1 v}-\bar{\Gamma}_{1 c}$ & $\bar{\Gamma}_{2 v}-\bar{\Gamma}_{1 c}$ & $\bar{\Gamma}_{3 v}-\bar{\Gamma}_{1 c}$ \\
\hline Energy (meV) & 2067 & 2136 & 2427 \\
\hline
\end{tabular}

Table 2: Inerband transition energies (in meV) of the corrugated superlattice

\begin{tabular}{|l|c|c|}
\hline Transition & $\bar{\Gamma}_{1 v}-\bar{\Gamma}_{2 c}$ & $\bar{\Gamma}_{2 v}-\bar{\Gamma}_{2 c}$ \\
\hline Energy (meV) & 1953 & 2011 \\
\hline
\end{tabular}

by:

The anisotropy rate of the two main polarization orientation for a given interband transition is defined

$$
\tau=\frac{I(0)-I\left(\frac{\pi}{2}\right)}{I(0)+I\left(\frac{\pi}{2}\right)}
$$

For the non-corrugated superlattice, the anisotropy rate for, respectively, $\bar{\Gamma}_{1 v}-\bar{\Gamma}_{1 c}, \bar{\Gamma}_{2 v}-\bar{\Gamma}_{1 c}$ and $\bar{\Gamma}_{3 v}-\bar{\Gamma}_{1 c}$ transitions are: $-0.096,0.172$ and 0.084 . We remark that the anisotropy rate is weak for these three interband transitions.

As regards the corrugated superlattice, the anisotropy rate for, respectively, $\bar{\Gamma}_{1 v}-\bar{\Gamma}_{2 c}$ and $\bar{\Gamma}_{2 v}-\bar{\Gamma}_{2 c}$ interband transitions are: -0.746 and 0.998 . In the opposite the anisotropy rate is strong for these two interband transitions.

For the two transitions $\bar{\Gamma}_{1 v}-\bar{\Gamma}_{2 c}$ and $\bar{\Gamma}_{1 v}-\bar{\Gamma}_{1 c}$, which originate from the heavy -hole-like states, the transition probability for light polarized along the [-2,3,3] direction is always larger than for the $[0,-1,1]$ one (more larger for the transition $\bar{\Gamma}_{1 v}-\bar{\Gamma}_{2 c}$ ). The opposite situation occurs for the second transitions $\bar{\Gamma}_{2 v}-\bar{\Gamma}_{2 c}$ and $\bar{\Gamma}_{2 v}-\bar{\Gamma}_{1 c}$ for which the initial valence state is light-hole-like.

For the $\bar{\Gamma}_{3 v}-\bar{\Gamma}_{1 c}$ transition which the initial valence state is split-off-like, the transition probability for the light polarized along the $[0,-1,1]$ direction is always larger than for the $[-2,3,3]$ one.

Our results reveal a decrease in the transition probability for the transitions from the heavy-hole-like states when the polarization angle $\theta$ increases from 0 to $\frac{\pi}{2}$. However we observe a increase in the transition probability with respect to the polarization angle for the transition from the light-hole and split-off-like states.

The significant result is that, for the non-corrugated and corrugated superlattices, the optical anisotropy is reduced when the light polarization pass from the $[-2,3,3]$ direction to $[0,-1,1]$ direction for the transitions from the heavy-hole-like states. The opposite situation occurs for the transition from the light-hole and split-offlike states. 


\section{Conclusions}

In summary, we have studied theoretically the electronic and optical properties of non-corrugated (311) $(\mathrm{GaAs})_{8} /(\mathrm{AlAs})_{8}$ superlattice and corrugated $(311)(\mathrm{GaAs})_{16} /(\mathrm{AlAs})_{16}$ superlattices, using the empirical tightbinding model with realistic parameters determined from the bulk bands. Then we have compared the optical anisotropy of corrugated and non-corrugated (311) superlattices

We have also calculated the optical properties for these heterostructures and we have compared their optical anisotropy. Thus we have found that, for non-corrugated (311) superlattice, the anisotropy rate is weak for the three interband transition $\bar{\Gamma}_{1 v}-\bar{\Gamma}_{1 c}, \bar{\Gamma}_{2 v}-\bar{\Gamma}_{1 c}$ and $\bar{\Gamma}_{3 v}-\bar{\Gamma}_{1 c}$. In the opposite the anisotropy rate is strong for the two interband transitions $\bar{\Gamma}_{1 v}-\bar{\Gamma}_{2 c}$ and $\bar{\Gamma}_{1 v}-\bar{\Gamma}_{1 c}$, which originate from the heavy -hole-like states.

The important result is that, for two types of the studied superlattices, the optical anisotropy is reduced when the light polarization pass from the $[-2,3,3]$ direction to $[0,-1,1]$ direction for the transitions from the heavy-hole-like states. The opposite situation occurs for the transition from the light-hole and split-off-like states. This shown the influence of the large in-plane anisotropy of the valence band states which modifies the strengths of the optical transitions and the role of the lateral confinement in determining the optical properties.

\section{References}

[1] X. Duan, Y. Huang, Y. Cui, J. Wang, and C.M. Lieber, Indium phosphide nanowires as building blocks for nanoscale electronic and optoelectronic devices, Nature 409, 2001, 66-69.

[2] R. Nötzel, N.N. Ledentsov, L. Däweritz, H. Hohenstein, and K. Ploog, Direct Synthesis of Corrugated Superlattices on Non-(100) Oriented Surfaces, Phys. Rev. Lett. 67(27), 1991, 3812-3815.

[3] R. Nötzel, N.N. Ledentsov, L. Däweritz, K. Ploog, and M. Hohenstein, Semiconductor quantum-wire structures directly grown on high-index surfaces, Phys. Rev. B 45(7), 1992, 3507-3515.

[4] D.A. Contreras-Solorio, V.R. Velasco, and J.F. Garcia-Moliner, Electronic structure of (311) A1As-GaAs superlattices, Phys. Rev. B 47(8), 1993, 4651-4654.

[5] A.B. Vorob'ev, A.K. Gutakovsky, V.Ya. Prinz, V.V. Preobrazhenskii, and M.A. Putyato, Interface corrugation in GaAs/AlAs (311)A superlattices, Appl. Phys. Lett. 77(19), 2000, 2976-2978.

[6] N.N. Ledentsov, D. Litvinov, A. Rosenauer, D. Gerthsen, I.P. Soshnikov, V.A. Shchukin, V.M. Ustinov, A.Yu. Egorov, A.E. Zhukov, V.A. Volodin, M.D. Efremov, V.V. Preobrazhenskii, B.P. Semyagin, D. Bimberg, and Zh.I. Alferov, Interface Structure and Growth Mode of Quantum Wire and Quantum Dot GaAs-AlAs Structures on Corrugated (311)A Surfaces, J. Electron. Mater. 30(5), 2001, 463-470.

[7] D. Litvinov, A. Rosenauer, D. Gerthsen, N.N. Ledentsov, D. Bimberg, G.A. Ljubas, V.V. Bolotov, V.A. Volodin, M.D. Efremov, V.V. Preobrazhenskii, B.R. Semyagin, and I.P. Soshnikov, Ordered arrays of vertically correlated GaAs and AlAs quantum wires grown on a GaAs(311)A Surface, Appl. Phys. Lett. 81(6), 2002, 1080-1082.

[8] S. Bednarek, B. Szafran, R.J. Dudek, and K. Lis, Induced quantum dots and wires: electron storage and delivery, Phys. Rev. Lett. 100, 2008, 126805-1-4.

[9] V. Pardo, and W.E. Pickett, Half-metallic semi-Dirac-point generated by quantum confinement in $\mathrm{TiO}_{2} / \mathrm{VO}_{2}$ nanostructures, Phys. Rev. Lett. 102, 2009, 166803-1-4.

[10] O. Bierwagen, and W.T. Masselink, Electronic switching device based on gate-controlled conductivity anisotropy, Appl. Phys. Lett. 90, 2007, 133507-1-3.

[11] H. Pettersson, J. Trangardh, A.I. Persson, L. Landin, D. Hessman, and L. Samuelson, Infrared photodetectors in heterostructures nanowires, Nano Lett. 6(2), 2006, 229-232.

[12] C.L. Tsai, C. Xu, K.C. Hsieh, and K.Y. Cheng, Growth optimization of InGaAs quantum wires for infrared photodetector applications, J. Vac. Sci. Technol. B 24(3), 2006, 1527-1531.

[13] A. Nasr, Performance of quantum wire infrared photodetectors under illumination conditions, Opt. Laser Technol. 41, 2009, 871876.

[14] P. Fei, P-H. Yeh, J. Zhou, S. Xu, Y. Gao, J. Song, Y. Gu, Y. Huang, and Z.L. Wang, Piezoelectric potential gated field-effect transistor based on a free-standing ZnO wire, Nano Lett. 9(10), 2009, 3435-3439.

[15] C.R. Müller, L. Worschech, and A. Forchel, Inversion of hysteresis in quantum dot controlled quantum-wire transistor, Phys. Rev. B 79, 2009, 205307-1-6.

[16] M.D. Kelzenberg, S.W. Boettcher, J.A. Petykiewicz, D.B. Turner-Evans, M.C. Putnam, E.L. Warren, J.M. Spurgeon, R.M. Briggs, N.S. Lewis, and H.A. Atwater, Enhanced absorption and carrier collection in Si wire arrays for photovoltaic applications, Nat. Mater. 9, 2010, 239-244.

[17] Y. Nishimoto, H. Yagi, K. Miura, D. Plumwongrot, K. Ohira, T. Maruyama, and S. Arai, High T 0 operation of $1590 \mathrm{~nm}$ GaInAs/InP quantum-wire distributed feedback lasers by Bragg wavelength detuning, Jpn. J. Appl. Phys. 46(17), 2007, L411-L413.

[18] V.G. Dorogan, Z.M. Wang, V.P. Kunets, M. Schmidbauer, Y.Z. Xie, M.D. Teodoro, P.M. Lytvyn, Y.I. Mazur, and G.J. Salamo, Alignment and optical polarization of InGaAs quantum wires on GaAs high index surfaces, Mater. Lett. 65, 2011, 1427-1430.

[19] C. Jouanin, A. Hallaoui, and D. Bertho, Optical anisotropy of (311) superlattices, Phys. Rev. B 50, $1994,1645-1648$.

[20] C. Jouanin, and D. Bertho, Electronic and optical properties of corrugated GaAs/AlAs superlattices, Superlattices Microstruct. 16 (3), 1994, 229-233.

[21] P. Vogl, H.P. Hjalmarson, and J.D. Dow, A semi-empirical tight-binding theory of the electronic structure of semiconductors, J. Phys. Chem. Solids 44(5), 1983, 365-378.

[22] C. Jouanin, and D. Bertho, Confined electron states in corrugated GaAs/AlAs superlattices, J. Phys. IV 3(C5), 1993, 287-290.

[23] C. Benoit, E. Royer, and G. Poussigue, The spectral moments method, J. Phys. Condens. Matter 4, $1992,3125-3152$. 